\title{
The Five-Pattern Personality Inventory (FPPI), a Chinese localized personality scale, relates to topological properties of resting-state brain networks
}

\author{
Xiaozhen Li \\ Shanxi Medical University; First hospital of Shanxi Medical University. \\ Yanfang Wang \\ Shanxi Medical University \\ Hao Guo
}

Taiyuan University of Technology

Jian Du

China Academy of Chinese Medical Sciences

Hao Wang

China Academy of Chinese Medical Sciences

Junjie Wang

First Hospital of Shanxi Medical University

Yao Li

Taiyuan University of Technology

Jing Li

First Hospital of Shanxi Medical University

Chao Sun

Taiyuan University of Technology

Qiuli Yang

China Academy of Chinese Medical Sciences

Xu Yong ( $\nabla$ xuyongsmu@vip.163.com)

First Hospital of Shanxi Medical University

\section{Research}

Keywords: resting-state fMRI, graph analysis, topological properties, Chinese personality traits, FivePattern Personality Inventory (FPPI)

Posted Date: April 30th, 2020 
DOI: https://doi.org/10.21203/rs.3.rs-23608/v1

License: (c) (i) This work is licensed under a Creative Commons Attribution 4.0 International License. Read Full License 


\section{Abstract}

Background Most studies assessing personality-brain associations have used Western personality questionnaires, which have been adapted for individuals in Western cultures. However, personality is influenced by culture and customs, and Western personality questionnaires may not objectively reflect the personality characteristics of individuals in Eastern cultures such as China. Hence, we adopted the graph analysis approach and the Chinese localized scale, the Five-Pattern Personality Inventory (FPPI), to explore the personality-brain associations of individuals in China.

Methods The functional connectivity among 90 specific regions of the brain was mapped using functional magnetic resonance imaging (fMRI) data obtained from 109 healthy adult Chinese subjects. This allowed for the construction of brain networks and the analysis of personality-brain associations.

Results In the present study, the Chinese personality-brain associations were traced to specific regions in the brain, including the frontal, temporal, and occipital cortices. Shao Yin scores and nodal metrics were associated with the brain regions of the executive control network and the salience network (SN), primarily in the left hemisphere. Characteristics of the Shao Yin and Shao Yang traits were consistent with the function of their corresponding correlated brain regions, which may supply evidence for the neurobiological basis of personality traits in Chinese culture.

Conclusion In summary, these results provide the brain mechanism foundations of personality traits in the FPPI. Furthermore, these findings provide a new perspective to help researchers understand personality-brain associations in the Chinese culture.

\section{Background}

The study of personality is at the core of clinical psychology. Personality refers to individual tendencies to express stable and specific patterns of cognition, emotion, and behavior [1]. Researchers have long believed that personality is a neurobiological product [2], yet there have been few advances in our understanding of the neural mechanisms of human personality during the past decade. In recent years, a growing number of studies examining brain-personality mechanisms have employed the Western personality-encompassing Eysenck Personality Questionnaire (EPQ) and NEO Personality Inventory and Tridimensional Personality Questionnaire (TPQ) for guidance [3-5]. However, personality is shaped and formed by an intricate interplay of biological, environmental, and experiential (i.e., cultural) factors and influences [6].

Whether the Western personality scales can objectively reflect the personality characteristics of people in East Asia, especially in China, is questionable. Many cross-cultural studies have revealed differences in common personality traits between Western and Eastern cultures, suggesting that a single questionnaire may not be feasible for assessing personality traits between two different populations [7, 8]. Therefore, geographical region-specific personality scales should be considered when conducting brain-personality studies across different cultures. 
The research titled "The yin and yang of neurotrophin action" by Lu has successfully introduced the Yin and Yang model of traditional Chinese culture to neuroscience [9]. Recently, the Five-Pattern Personality Inventory (FPPI) has become more popular for studying personality in East Asia. The FPPI originates from the Chinese philosophy of Yin and Yang and its five principles of Tai Yang, Shao Yang, Ying Yang, Shao Yin, and Tai Yin. These principles are used to characterize various aspects of social behavior and emotional responsiveness [10]. Our previous study have revealed the mechanism differences of typical yin and typical yang trait [11]. In addition, the FPPI is referred to as the Eastern version of the EPQ [12]. And our group have also found the Chinese five-pattern personality traits have a close relationship with Eysenck's personality traits and that both may be engaged in similar neurobiological mechanisms to some extent [13]. These two scales, which are classified as biological perspective scales, both assume that personality is a neurobiological product and concentrate on the relationships between personality traits and neurobiological mechanisms.

In the current study, we aimed to show that inter-individual differences in the personality traits of Chinese individuals assessed by FPPI could be associated with the topological characteristics of functional brain networks. To accomplish this goal, we utilized the graph theory to identify the intrinsic functional connectivity mechanisms associated with personality traits using the resting-state fMRI data of 109 healthy Chinese adult subjects.

\section{Materials And Methods}

\subsection{Participants}

All participants were recruited by bulletin advertisements in the Shanxi province of China. The exclusion criteria for this study included subjects having neurological or psychiatric diseases, cognitive disabilities, contraindications to imaging studies, history of substance abuse (i.e., illicit drugs or alcohol), or any other major medical illnesses. After applying the exclusion criteria, the remaining 109 subjects were included in this study, consisting of 24 males and 85 females with an average age of $24.8 \pm 2.4$ years (range: $21-$ 33 years). The subjects provided written informed consent before the study and were reimbursed for their time. Approval for this study was obtained from the Shanxi Medical University Ethics Committee.

\subsection{Personality assessment}

The FPPI consists of 103 "yes" (score = 1) or "no" (score = 0 ) items. These questions assess personality in terms of five different traits: Tai Yang, Shao Yang, Yin Yang, Shao Yin, and Tai Yin. In addition, "lie," which includes 8 items, is another personality dimension used to measure the truthfulness of subject responses. If the score on this dimension was $>4$, the questionnaire was regarded as invalid and excluded from the study. The FPPI has shown good reliability, construct validity, and convergent validity with other personality scales [14].

\subsection{Data acquisition}


All fMRI images were collected on a 3.0T Siemens Trio scanner (Siemens Healthcare GmbH, Erlangen, Germany) at Shanxi Provincial People's Hospital. The standard eight-channel phase-array head coil was employed, and image artifacts, such a head motion and scanner noise, were reduced with foam padding and headphones. The images were transversely collected using the echo-planar sequence with the following protocol: 32 slices, TR of $2,500 \mathrm{msec}$, TE of $40 \mathrm{msec}$, FA of $90^{\circ}$, matrix of $64 \times 64$, voxel size of $3 \mathrm{~mm} \times 3 \mathrm{~mm} \times 3 \mathrm{~mm}$, FOV of $240 \mathrm{~mm} \times 240 \mathrm{~mm}$, and 212 volume.

\subsection{Image pre-processing}

The fMRI data were preprocessed with the SPM12 toolset (http://www.fil.ion.ucl.ac.uk/spm). Due to signal instability and subject acclimation, the initial 10 volumes were dismissed. For the 202 images that remained, corrections were implemented to account for delays in slices and head movement. The translational parameters were greater than allowable $(2.5 \mathrm{~mm})$ in five datasets. After excluding these five datasets, the remaining images were normalized with respect to the traditional SPM8 echo-planar image template and resampled to $3 \mathrm{~mm}$ cubic voxels. Next, spatial smoothing was applied (4 $\mathrm{mm}$ full width at half maximum [FWHM] Gaussian kernel), and the linear trends were removed from the final images. Lastly, we regressed the cerebrospinal fluid (CSF), white matter, and six head movement parameters.

\subsection{Computation of functional connectivity networks}

Anatomical parcellation was accomplished with automated anatomical labeling (AAL) by separating the images into 45 regions of interest (ROI) for each hemisphere of the brain, which resulted in 90 total ROI. Edges of the network were defined by generating $90 \times 90$ correlation matrixes and calculating the regionwise Pearson's correlation coefficients. The threshold was set as the sparsity (S), or the ratio of actual existing edges divided by the maximum possible edges in the network. The construction of the brain networks is shown in Fig. 1.

Insert Fig. 1 here

\subsection{Graph theoretical analysis: Network metrics}

The topological features of the brain functional networks were assessed by the nodal and global network measures. First, the global metrics, consisting of the normalized clustering coefficient $(\mathrm{\gamma})$ and characteristic path length $(\lambda)$, were computed. In comparison to the random networks with low clustering coefficients and shorter path lengths, small-world networks display higher clustering coefficients and similar path lengths (i.e., $Y=C p / C r a n d ~>1, \lambda=L p / L$ rand $\approx 1$ ) [15]. Small-worldness is the quantitative measurement that includes the combination of these two conditions (i.e., $\sigma=\gamma / \lambda>1$ ) [16]. Meanwhile, the nodal measures encompassed Freeman's betweenness centrality $(B C I)$ and degree $(K i)$. More details on the mathematical definitions and interpretations of the network metrics may be found in the Supplementary Material.

\subsection{Associations between the network metrics and personality traits}


All of the nodal measures reached the threshold in the range of $0.05 \leq T \leq 0.40$ (interval $=0.01$ ). The area under the curve (AUC) was calculated for each network metric in order to obtain a summarized scalar for topological characterization that was independent of the single-threshold selection [17]. A partial correlation was determined between each of the five trait scores and the AUC of each network metric, with age, gender, and education (years) as covariates. The false discovery rate (FDR) was applied to the data for correction [18] using the code developed by Groppe in 2010 with Matlab (MathWorks, Natick, MA, USA).

\subsection{Statistical analysis}

$P$-values $<0.05$ were considered statistically significant.

\section{Results}

\subsection{Descriptive statistics of the personality traits}

The demographic and personality data of the participants are shown in Table $1(n=109$; males $=24)$. The corresponding correlation analysis is shown in Table 2. There were both negative and positive correlations found among the five personality traits. The next goal was to determine the effects that were uniquely driven by each personality trait. Thus, the four remaining personality traits were included as covariates for computing the partial correlation between the AUC of the network metrics and each of the personality traits.

Table 1

Demographic and personality characteristics of participants $(n=109)$.

\begin{tabular}{|llll|}
\hline Measure & Mean & $S D$ & Range \\
\hline Age & 24.75 & 2.358 & $21-33$ \\
\hline Education (years) & 17.45 & 1.500 & $16-19$ \\
\hline Tai Yang & 9.84 & 3.824 & $1-18$ \\
\hline Shao Yang & 11.48 & 3.955 & $2-20$ \\
\hline Yin Yang & 6.01 & 2.311 & $0-10$ \\
\hline Shao Yin & 13.14 & 3.026 & $7-21$ \\
\hline Tai Yin & 8.81 & 5.234 & $0-20$ \\
\hline
\end{tabular}


Table 2

Correlations between the five personality types.

\begin{tabular}{|llllll|}
\hline & Tai Yang & Shao Yang & Yin Yang & Shao Yin & Tai Yin \\
\hline Tai Yang & 1 & & & & \\
\hline Shao Yang & $.464^{* \star}$ & 1 & & & \\
\hline Yin Yang & $.196^{*}$ & .186 & 1 & & \\
\hline Shao Yin & .000 & -.130 & $.453^{* \star}$ & 1 & 1 \\
\hline Tai Yin & $-.266^{* *}$ & $-.366^{* *}$ & $-.498^{* *}$ & .086 & 1 \\
\hline${ }^{*} p<0.05$. & & & & & \\
\hline${ }^{* *} p<0.01$. & & & & & \\
\hline
\end{tabular}

Insert Table 1 and Table 2 here

\subsection{Associations between the personality traits and nodal metrics}

Those personality traits and nodal metrics that showed strong positive correlations are shown in Table 3 . We found that Tai Yang scores were positively associated with BCi in CUN.L $(p=0.001)$, as shown in Fig. 2a. Positive associations were found between Shao Yang scores and $B C i$ in PCL.L $(p=0.030)$ and PCL.R $(p=0.005)$, as shown in Fig. $2 \mathrm{~b}$ and Fig. $2 \mathrm{c}$, respectively. Correlations between $B C i$ and Shao Yin scores were found in ACG.L $(p=0.024)$ and INS.L $(p=0.017)$, as shown in Fig. 3a. In addition, the associations between Ki and Shao Yin scores in ACG.L $(p=0.017)$, INS.L $(p=0.017)$, CAU.L $(p=0.009)$, and CAU.R $(p=0.017)$ are shown in Fig. 3b. 
Table 3

Correlations between personality traits and nodal metrics

\begin{tabular}{|c|c|c|c|c|c|c|}
\hline & Personality trait & Nodal metrics & Brain region & Side & $r$ & $\begin{array}{l}\text { Adjusted } \\
p \text {-value }\end{array}$ \\
\hline 1 & Tai Yang & $B C_{i}$ & CUN & $\mathrm{L}$ & 0.369 & 0.001 \\
\hline 2 & Shao Yang & $B C_{i}$ & PCL & $\mathrm{L}$ & 0.213 & 0.030 \\
\hline 3 & & $B C_{i}$ & PCL & $\mathrm{R}$ & 0.313 & 0.005 \\
\hline 4 & Shao Yin & $B C_{i}$ & ACG & $L$ & 0.225 & 0.024 \\
\hline 5 & & $B C_{i}$ & INS & L & 0.242 & 0.017 \\
\hline 6 & Shao Yin & $K_{i}$ & ACG & L & 0.252 & 0.017 \\
\hline 7 & & $K_{i}$ & INS & $\mathrm{L}$ & 0.247 & 0.017 \\
\hline 8 & & $K_{i}$ & CAU & $L$ & 0.284 & 0.009 \\
\hline 9 & & $K_{i}$ & CAU & $\mathrm{R}$ & 0.248 & 0.017 \\
\hline \multicolumn{7}{|c|}{$\begin{array}{l}\text { Abbreviations: BCi and Ki represent the betweenness centrality and the degree, respectively. There are } \\
\text { two nodal metrics. Brain regions are from } 90 \text { ROI. CUN: cuneus; PCL: paracentral lobule; ACG: anterior } \\
\text { cingulate and paracingulate gyri; INS: insula; CAU: caudate nucleus; L: left; R: right. r represents the } \\
\text { partial correlation coefficients between the nodal metrics and personality traits, and p represents the } \\
\text { significance level after correcting for multiple comparisons. }\end{array}$} \\
\hline
\end{tabular}

Insert Table 3, Fig. 2 and Fig. 3 here

\section{Discussion}

We have shown that personality traits and brain topological properties are associated with both global and regional metrics. Our findings showed obvious small-world properties of the functional brain networks, which is in accordance with several previous studies [19]. Meanwhile, the personality-brain associations in the Chinese participants were found in specific regions of the frontal, temporal, and occipital cortex. Compared with previous Western personality-brain correlation studies, we found that the personality traits of Chinese and Western individuals show both similarities and differences in brain mechanisms. Furthermore, these findings provide a new perspective for researchers to better understand the personality-brain associations in Chinese culture.

\subsection{Functional brain correlates of the Shao Yin trait}

The Shao Yin is a trait reflecting restrained behavior, toughness, and alertness to the surroundings. While topological correlates were observed for Tai Yang and Shao Yang, the Shao Yin trait showed the 
strongest connection with brain function, including positive correlations with the normalized clustering coefficient on the global metrics and regional function of brain areas in the salience network (SN) and executive control network (one of the sub-networks of attention). These two networks are involved in the processing and integration of a variety of external cognitive and emotional information $[20,21]$. The executive control network, which encompasses the anterior cingulate gyrus, supplementary motor area, and caudate, is thought to be associated with conflict resolution abilities and the inhibition of automatic responses [22]. The SN, which comprises the anterior cingulate cortex and insula, is involved in the allocation of attention and segregation of internal and external cognitive data [23]. Meanwhile, the SN also functions as the biological switch between the task-related and self-monitoring networks [24].

Herein, the function of these two networks was consistent with the Shao Yin trait feature of restrained behavior and alertness to one's surroundings, which suggests a rapid shift from awareness to consciousness. This indicates that the Shao Yin trait, which is the central characteristic of Chinese personality ( $\mathrm{Li}$ et al. 2014), is related to most functional variables of the brain. In addition, several studies have shown positive associations between neuroticism in the Eysenck Personality Questionnaire (EPQ) and three brain regions (ACG, INS, and CAU) via resting-state fMRI [25-27], task fMRI [28, 29], and positron emission tomography (PET) [30]. Therefore, we believe that Shao Yin and neuroticism have similar brain mechanisms.

The left hemisphere of the brain showed the strongest correlations in our study, indicating the lateralization of regions within the left hemisphere with the Shao Yin trait. While the left hemisphere is primarily involved in social approach, the right hemisphere is preferentially associated with social withdrawal in personality [31]. A number of studies have revealed interventions that increase intrinsic alertness, resulting in leftward-moving spatial biases [32, 33]. Thus, the alertness of Shao Yin could be the intrinsic motivation of "approach." It would be over-simplistic to associate every aspect of the Shao Yin trait with the left hemisphere, so further studies are needed to explore the restrained characteristics of Shao Yin and lateralization.

\subsection{Functional brain correlates of the Tai Yang and Shao Yang trait}

Tai Yang, which is a trait reflecting impulsive, aggressive, and adventurous behaviors, is positively correlated with nodal metrics in the left cuneus (CUN.L). CUN is believed to play an essential role in cognitive processing [34] and risk-taking actions. For instance, previous fMRI studies have shown that risk-taking actions elicit significantly stronger activation of the CUN [35, 36]. Moreover, CUN activation was found to be higher in aggressive veterans compared with the general population, which suggests a close connection between CUN and aggression [37]. We infer that CUN appears to subserve aggressive and risk-taking actions, aspects commonly associated with Tai Yang. In addition, the link between CUN and extroversion or extroversion-related traits has been well established [38, 39]. The Shao yang trait, which reflects the tendency toward more movement of the body and physical activity, is positively associated with nodal metrics in the bilateral paracentral lobule (PCL). PCL is regarded as the 
continuation of the precentral and postcentral gyrus and is known to regulate the motor and somatosensory representation of the foot and leg [40,41]. PCL may subserve the movement of the body, a trait commonly related to Shao Yang.

\section{Limitations}

This study has two limitations that should be addressed in future work. First, the neurobiological mechanisms of only three of the five personality traits were discovered. Unfortunately, we were unable to elucidate the mechanisms of the Yin Yang and Tai Yin personality traits. This could have been due to our small sample size, and future studies using larger populations may provide more insight into this topic. Second, the molecular mechanism involved in the connection between Chinese personality traits and structural brain networks remains unknown, yet future studies using diffusion tensor imaging (DTI) may provide new insights.

\section{Conclusions}

In summary, the present study uncovered strong associations between Chinese personality traits and brain topological properties based on the commonly utilized FPPI. This was accomplished by applying graph theoretical analysis to the data from the resting-state fMRI. These results offer a new perspective for our understanding of personality-brain associations in the Chinese culture.

\section{Abbreviations}

\section{ACG}

anterior cingulate and paracingulate gyri

AUC

area under the curve

$\mathrm{BCi}$

betweenness centrality

CAU

caudate nucleus

CSF

cerebrospinal fluid

CUN

cuneus

DTI

diffusion tensor imaging

EPQ

Eysenck personality questionnaire

\section{fMRI}

functional magnetic resonance imaging 
FPPI

Five-pattern personality inventory

\section{FWHM}

full width at half maximum

INS

insula

$\mathrm{Ki}$

degree

PCL

paracentral lobule

ROI

regions of interest

SN

salience network

TPQ

Tridimensional Personality Questionnaire

\section{Declarations}

\section{Ethics approval and consent to participate}

The subjects provided written informed consent before the study and were reimbursed for their time. Approval for this study was obtained from the Shanxi Medical University Ethics Committee.

\section{Consent for publication}

Not applicable.

\section{Availability of data and materials}

The datasets analyzed during the current study are available from the corresponding author on reasonable request.

\section{Competing interests}

The authors declare that they have no competing interests.

\section{Funding}

This work was supported by the National Key Research and Development Program of China (2016YFC1307004; 2016YFC1307103), the National Natural Science Foundation of China (81571319; 61876124), the Multidisciplinary Team for Cognitive Impairment of the Shanxi Science and Technology Innovation Training Team (201705D131027), and the Support Program of the Youth Sanjin Scholars. 


\section{Authors' contributions}

$\mathrm{XY}$ and YQ designed and supervised the study. LX, WY, and GH drafted the manuscript. DJ, WH, WJ, LY and LJ undertook the statistical analyses and reviewed the literature. SC participated in data processing.

\section{Acknowledgements}

For the preprocessing and analysis of fMRI data, we thank Beijing Bu-Qi Science and Technology Development Co., Ltd. We also would like to thank the Department of Psychiatry at Shanxi Medical University and the Department of MR at Shanxi Province People's Hospital for their support.

\section{References}

1. Kim, S.H., Resting brain metabolic correlates of neuroticism and extraversion in young men. Neuroreport, 2008. 19: p. 883-886.

2. Nostro, A.D., V.I. Müller, A.T. Reid, and S.B. Eickhoff, Correlations Between Personality and Brain Structure: A Crucial Role of Gender. Cerebral Cortex, 2017. 27(7): p. 3698-3712.

3. Gao, Q., Q. Xu, X. Duan, W. Liao, J. Ding, Z. Zhang, Y. Li, G. Lu, and H. Chen, Extraversion and neuroticism relate to topological properties of resting-state brain networks. Frontiers in Human Neuroscience, 2013. 7(24): p. 257.

4. Lei, X., C. Chen, F. Xue, Q. He, C. Chen, Q. Liu, R.K. Moyzis, G. Xue, Z. Cao, and J. Li, Fiber connectivity between the striatum and cortical and subcortical regions is associated with temperaments in Chinese males. Neuroimage, 2014. 89(3): p. 226-234.

5. Li, T., X. Yan, Y. Li, J. Wang, Q. Li, H. Li, and J. Li, Neuronal Correlates of Individual Differences in the Big Five Personality Traits: Evidences from Cortical Morphology and Functional Homogeneity. Frontiers in Neuroscience, 2017. 11.

6. Mcadams, D.P. and J.L. Pals, A new Big Five: fundamental principles for an integrative science of personality. Am Psychol, 2006. 61(3): p. 204-217.

7. Singelis, T.M., H.C. Triandis, D.P.S. Bhawuk, and M.J. Gelfand, Horizontal and Vertical Dimensions of Individualism and Collectivism: A Theoretical and Measurement Refinement. Cross-Cultural Research: The Journal of Comparative Social Science, 1995. 29(3): p. 240-275.

8. Wang, D.F. and H. Cui, Theoretical and Empirical Comparison between Chinese and Western Personality Structure. Journal of Peking University, 2003.

9. Lu, B., P.T. Pang, and N.H. Woo, The yin and yang of neurotrophin action. Nat Rev Neurosci, 2005. 6(8): p. 603-14. 
10. Yang, Q., Xue, C., Personality theory and fifive-pattern personality inventory of TCM psychology, Chin. J. Basic Med. Sci. 12 (10) (2006) 777-779.

11. Li, X., C. Zhuo, H. Guo, J. Du, H. Wang, J. Wang, J. Li, W. Zhao, Y. Li, C. Sun, J. Zhang, Q. Yang, and Y. $\mathrm{Xu}$, Mechanism differences between typical yin and typical yang personality individuals assessed by Five-Pattern Personality Inventory (FPPI): Evidence from resting-state brain functional networks. Neurosci Lett, 2020. 718: p. 134745.

12. Ren, H., Applicability of Five-Pattern Personality Inventory (FPPI) to measure personality of college students - a comparative study with EPQ. Health vocational education, 2014. 32(12): p. 117-119.

13. Zhao, W., L. Song, J. Du, X. Li, H. Wang, L. Cheng, J. Li, L. Zhang, X. Li, Q. Yang, and Y. Xu, The Similarity Between Chinese Five-Pattern and Eysenck's Personality Traits: Evidence From Theory and Resting-State fMRI. Frontiers in Human Neuroscience, 2020. 14.

14. Zhang, Z, Structural Validity Analysis of the Five-Pattern Personality Inventory, The master dissertation of Shandong University of Traditional Chinese Medicine, 2013..

15. Watts, D.J. and S.H. Strogatz, Collective dynamics of 'small-world' networks. Nature, 1998. 393: p. 440.

16. Humphries, M.D. and K. Gurney, Network 'small-world-ness': a quantitative method for determining canonical network equivalence. Plos One, 2008. 3(4): p. e0002051.

17. Zhang, J., J. Wang, Q. Wu, W. Kuang, X. Huang, Y. He, and Q. Gong, Disrupted brain connectivity networks in drug-naive, first-episode major depressive disorder. Biological Psychiatry, 2011. 70(4): p. 334-342.

18. Benjamini, Y. and Y. Hochberg, Controlling the False Discovery Rate: A Practical and Powerful Approach to Multiple Testing. Journal of the Royal Statistical Society, 1995. 57(1): p. 289-300.

19. Moussa, M.N., C.D. Vechlekar, J.H. Burdette, M.R. Steen, C.E. Hugenschmidt, and P.J. Laurienti, Changes in Cognitive State Alter Human Functional Brain Networks. Frontiers in Human Neuroscience, 2011. 5(12): p. 83.

20. Pessoa, L., Attention and emotion. Annual Review of Biomedical Sciences, 2010. 10: p. 84-104.

21. Menon, V. and L.Q. Uddin, Saliency, switching, attention and control: a network model of insula function. Brain Structure \& Function, 2010. 214(5-6): p. 655-667.

22. Berger, A. and M.I. Posner, Pathologies of brain attentional networks. Neuroscience \& Biobehavioral Reviews, 2000. 24(1): p. 3-5.

23. Seeley, W.W., V. Menon, A.F. Schatzberg, J. Keller, G.H. Glover, H. Kenna, A.L. Reiss, and M.D. Greicius, Dissociable intrinsic connectivity networks for salience processing and executive control. Journal of Neuroscience, 2007. 27(9): p. 2349-2356.

24. Palaniyappan, L. and P.F. Liddle, Does the salience network play a cardinal role in psychosis? An emerging hypothesis of insular dysfunction. J Psychiatry Neurosci, 2012. 37(1): p. 17-27. 
25. Gao, Q., Q. Xu, Z. Long, X. Duan, W. Liao, J. Ding, Z. Zhang, Y. Li, G. Lu, and H. Chen, Extraversion and neuroticism relate to topological properties of resting-state brain networks. Frontiers in Human Neuroscience, 2013. 7(257).

26. Madsen, K.S., T.L. Jernigan, and W.F.C. Baaré, Neuroticism is associated with cingulum asymmetry in children: Opposite effects in boys and girls. 2013.

27. Mitchell, R.L.C. and V. Kumari, Hans Eysenck's interface between the brain and personality: Modern evidence on the cognitive neuroscience of personality. Personality \& Individual Differences, 2016. 103: p. 74-81.

28. Brühl, A.B., M.C. Viebke, T. Baumgartner, T. Kaffenberger, and U. Herwig, Neural correlates of personality dimensions and affective measures during the anticipation of emotional stimuli. Brain Imaging \& Behavior, 2011. 5(2): p. 86-96.

29. Feinstein, J.S., M.B. Stein, and M.P. Paulus, Anterior insula reactivity during certain decisions is associated with neuroticism. Social Cognitive \& Affective Neuroscience, 2006. 1(2): p. 136-142.

30. Fischer, H., G. Wik, and M. Fredrikson, Extraversion, neuroticism and brain function: A pet study of personality. Personality and Individual Differences, 1997. 23(2): p. 345-352.

31. Davidson, R.J., Anxiety and affective style: role of prefrontal cortex and amygdala. Biological Psychiatry, 2002. 51(1): p. 68-80.

32. Sheppard, D., J. Bradshaw, J. Mattingley, and P. Lee, Effects of stimulant medication on the lateralisation of linebisection judgements of children with attention deficit hyperactivitydisorder. $J$ Neurol Neurosurg Psychiatry, 1999. 66(1): p. 57-63.

33. Linnell, K.J., S. Caparos, and J. Davidoff, Urbanization increases left-bias in line-bisection: an expression of elevated levels of intrinsic alertness? Frontiers in Psychology, 2014. 5: p. 1127.

34. Mathias, B., J. Emanuel, R.E. Beaty, F. Andreas, K. Karl, and A.C. Neubauer, Brain mechanisms associated with internally directed attention and self-generated thought. Sci Rep, 2016. 6: p. 22959.

35. Bradley, M.M., D. Sabatinelli, P.J. Lang, J.R. Fitzsimmons, W. King, and P. Desai, Activation of the visual cortex in motivated attention. Behavioral Neuroscience, 2003. 117(2): p. 369-380.

36. Tamura, M., Y. Moriguchi, S. Higuchi, A. Hida, M. Enomoto, J. Umezawa, and K. Mishima, Neural Network Development in Late Adolescents during Observation of Risk-Taking Action. PLOS ONE, 2012. 7(6): p. e39527.

37. Heesink, L., T.E. Gladwin, D. Terburg, J.v. Honk, R. Kleber, and E. Geuze, Proximity alert! Distance related cuneus activation in military veterans with anger and aggression problems. Psychiatry Research: Neuroimaging, 2017. 266.

38. O'Gorman, R.L., V. Kumari, S.C.R. Williams, F.O. Zelaya, S.E.J. Connor, D.C. Alsop, and J.A. Gray, Personality factors correlate with regional cerebral perfusion. Neurolmage, 2006. 31(2): p. 489-495.

39. Ruffle, J.K., A.D. Farmer, M. Kano, V. Giampietro, Q. Aziz, and S.J. Coen, The influence of extraversion on brain activity at baseline and during the experience and expectation of visceral pain. Personality and Individual Differences, 2015. 74: p. 248-253. 
40. Laokaim, N.P., L. Fonville, V.P. Giampietro, S.C. Williams, A. Simmons, and K. Tchanturia, Aberrant function of learning and cognitive control networks underlie inefficient cognitive flexibility in anorexia nervosa: a cross-sectional fMRI study. Plos One, 2015. 10(5): p. e0124027.

41. Han, D., M. Li, M. Mei, and X. Sun, The functional and structural characteristics of the emotion network in alexithymia. Neuropsychiatr Dis Treat, 2018. 14: p. 991-998.

\section{Figures}
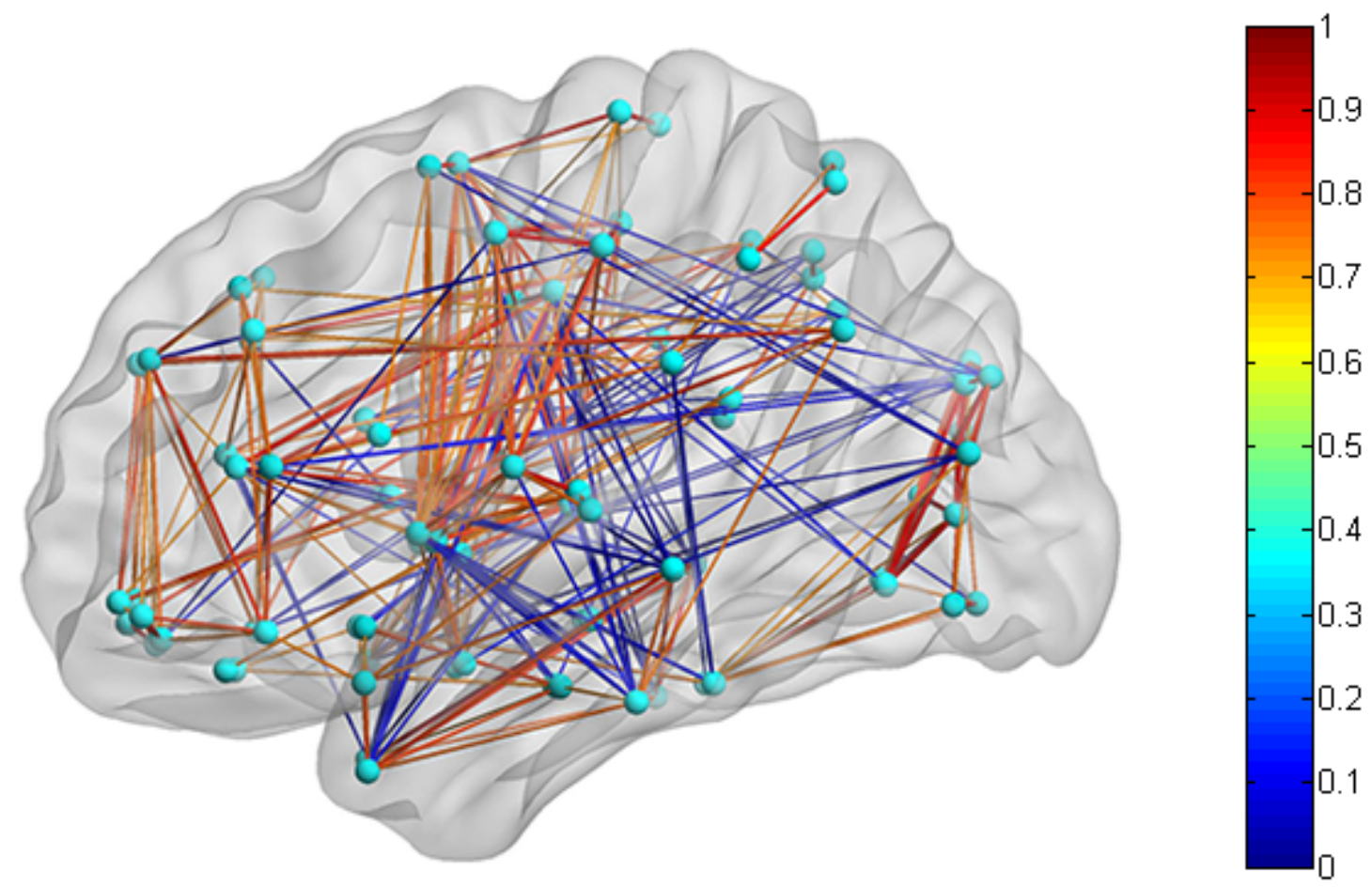

\section{Figure 1}

The networks construction. The connections of $90 \mathrm{ROI}$ in AAL are shown in the brain with the cyan blue dots reflecting the nodes, while the lines are indicative of neural connections between the nodes. The line colors are indicative of connection strength (scale from 0 to 1). ROI: regions of interest; AAL: automated anatomical labeling. 
(a)
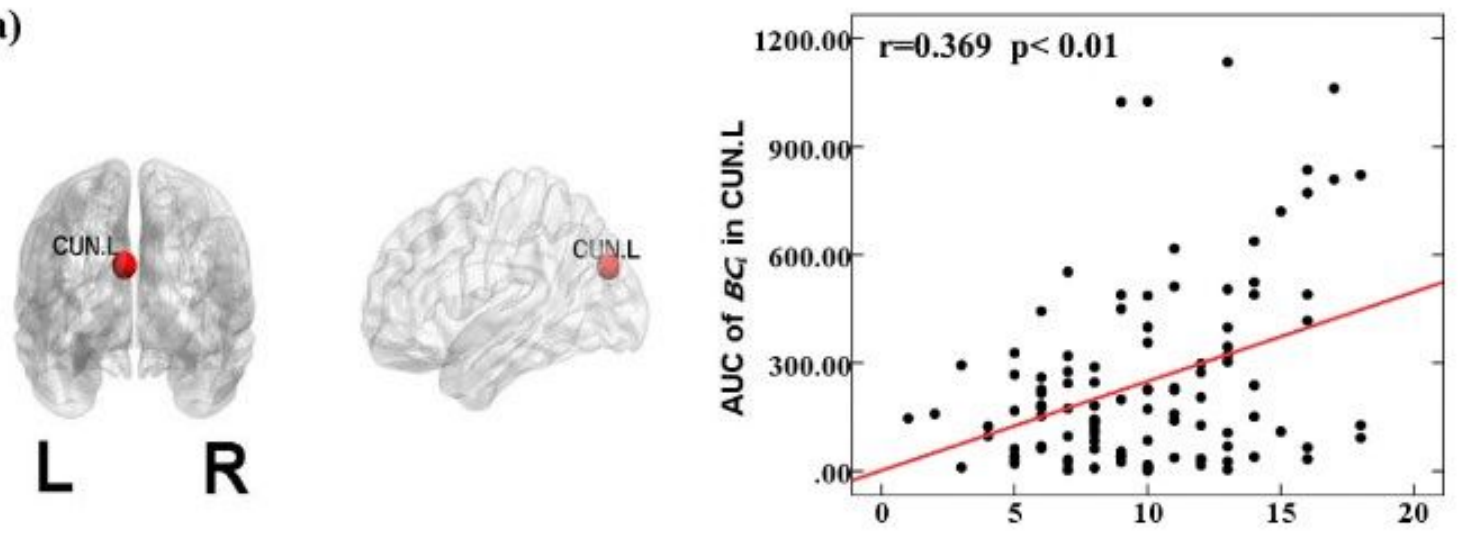

Tai Yang scores

(b)
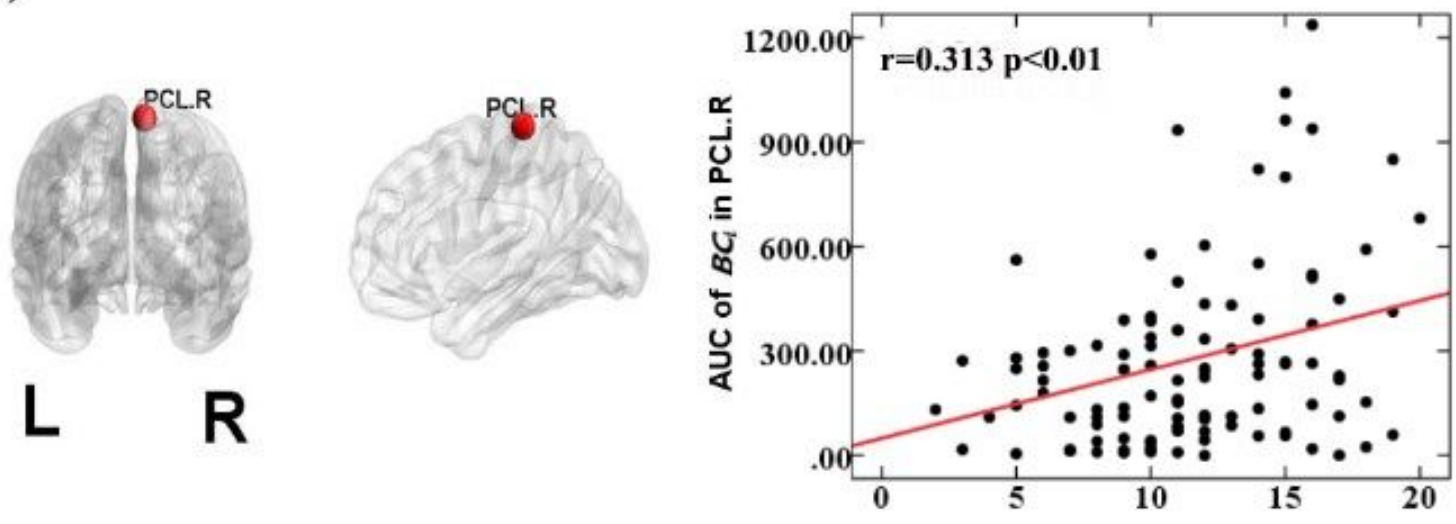

Shao Yang scores

(c)
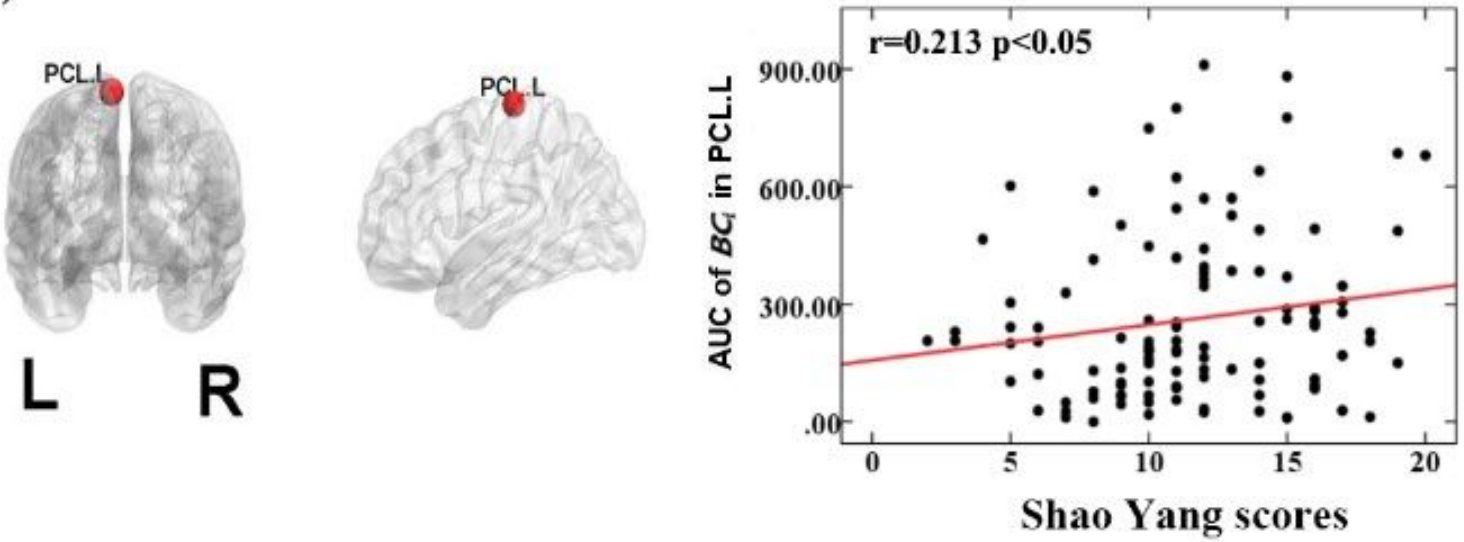

Figure 2

Correlations between the AUC of betweenness centrality (BCi) in the nodal metrics and Tai Yang scores. A strong positive correlation was detected between the AUC of BCi in CUN.L and the Tai Yang scores. In addition, the AUC of BCi in the bilateral PCL was positively correlated with Shao Yang scores. AUC was computed over the range of $0.05 \leq \mathrm{T} \leq 0.40$ (interval $=0.01$ ). CUN: cuneus; $P C L$ : paracentral lobule; $L$ : left. R: right. 

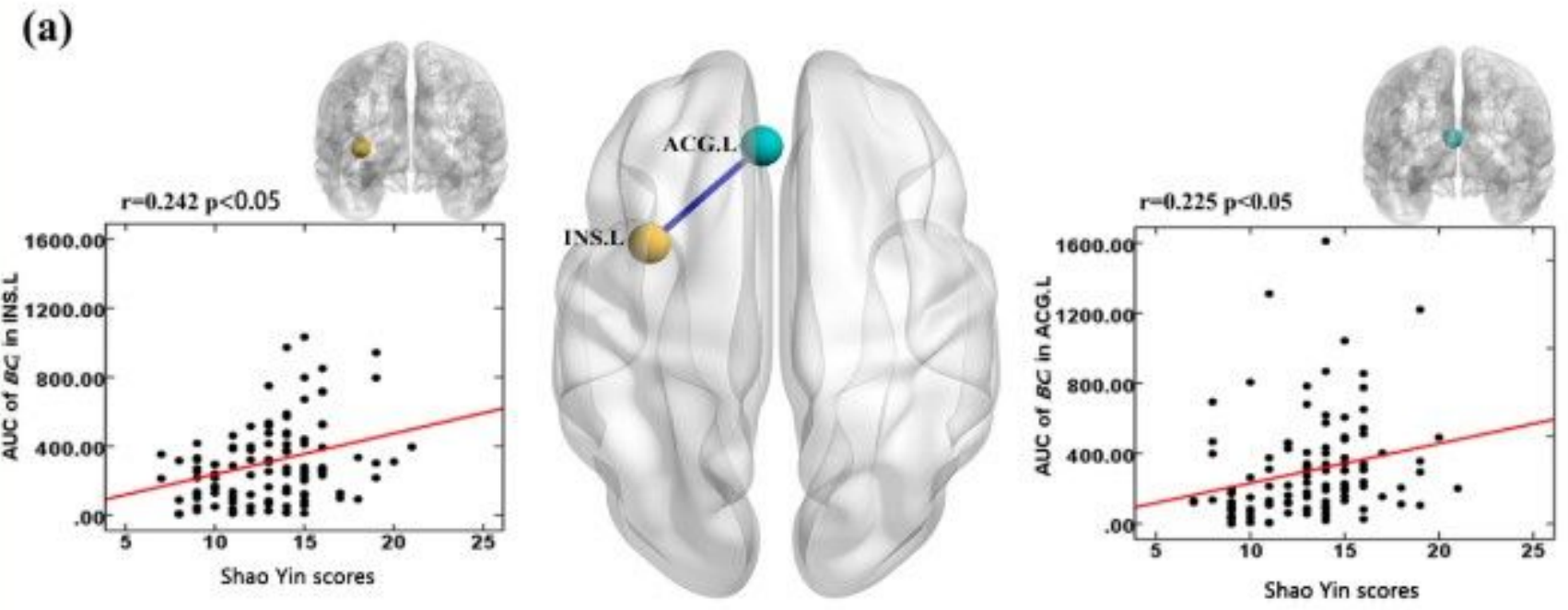

Associations between $B C i$ and Shao Yin scores
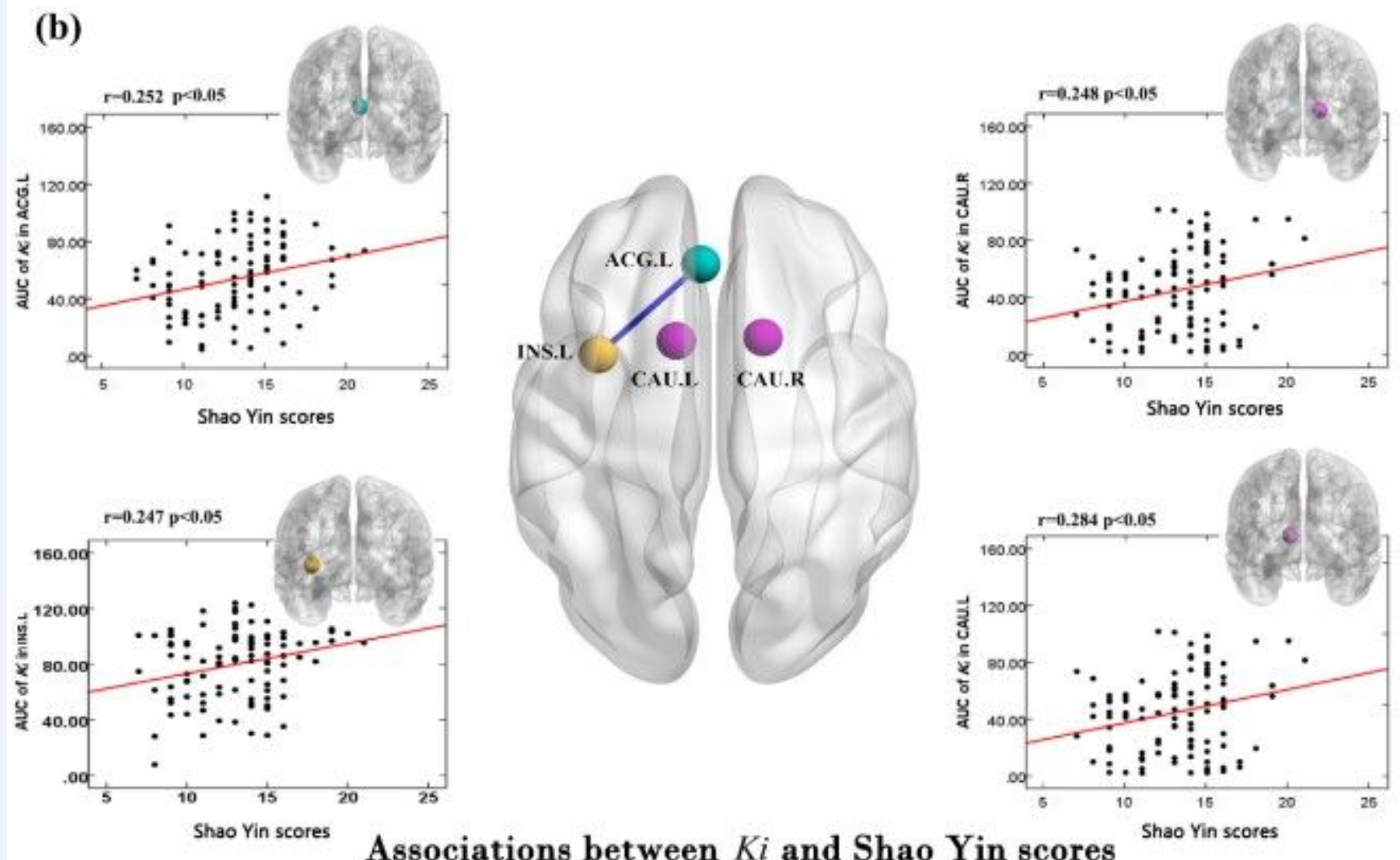

Associations between $K i$ and Shao Yin scores

Figure 3

Associations between the nodal metrics ( $\mathrm{BCi}$ and $\mathrm{Ki}$ ) and Shao Yin scores. Shao Yin scores were positively related to the AUC of BCi in ACG.L and INS.L. Positive associations were found between $\mathrm{Ki}$ and Shao Yin scores in ACG.L, INS.L, CAU.L, and CAU.R. The AUC was calculated over the range of $0.05 \leq \mathrm{T} \leq$ 0.40 (interval $=0.01$ ). The blue edge connects the brain regions in the salience network, while the gray edges connect the brain regions in the executive control network. ACG: anterior cingulate and paracingulate gyri; INS: insula; CAU: caudate nucleus; L: left; R: right. 


\section{Supplementary Files}

This is a list of supplementary files associated with this preprint. Click to download.

- wutaixusupplementarymaterial.docx 